Review

\title{
Genome Editing Using Mammalian Haploid Cells
}

\section{Takuro Horii and Izuho Hatada *}

Laboratory of Genome Science, Biosignal Genome Resource Center, Institute for Molecular and Cellular Regulation, Gunma University, 3-39-15 Showa-machi, Maebashi, Gunma 371-8512, Japan; E-Mail: horii@gunma-u.ac.jp

* Author to whom correspondence should be addressed; E-Mail: hatada@gunma-u.ac.jp; Tel.: +81-27-220-8057; Fax: +81-27-220-8110.

Academic Editor: Mark Richter

Received: 14 August 2015 / Accepted: 28 September 2015 / Published: 1 October 2015

\begin{abstract}
Haploid cells are useful for studying gene functions because disruption of a single allele can cause loss-of-function phenotypes. Recent success in generating haploid embryonic stem cells (ESCs) in mice, rats, and monkeys provides a new platform for simple genetic manipulation of the mammalian genome. Use of haploid ESCs enhances the genome-editing potential of the CRISPR/Cas system. For example, CRISPR/Cas was used in haploid ESCs to generate multiple knockouts and large deletions at high efficiency. In addition, genome-wide screening is facilitated by haploid cell lines containing gene knockout libraries.
\end{abstract}

Keywords: haploid; embryonic stem cell; CRISPR/Cas

\section{Introduction}

Ploidy refers to the number of sets of chromosomes in a cell. Diploid, meaning two chromosome sets, is the most common ploidy in animals. Diploid animals generally produce haploid gametes: for example, a mouse (M. musculus) has two genome sets $(2 n=40)$ in somatic cells, and generates haploid $(n=20)$ sperm and oocytes. However, these haploid cells are generally limited in their life cycle. For genetic analysis, haploid cells have several advantages over diploid cells for the study of gene function. In diploid cells, heterozygous mutations often lead to no phenotypic changes because a functional allele on a second chromosome set can mask the effects of the disruption of the same allele 
on the first chromosome set (Figure 1). Genetic analysis in diploid cells is therefore complex, and before genome editing technologies, such as CRISPR/Cas, became available, the analyses had to be performed either by selecting "targeted" clones undergoing loss-of-heterozygosity or sequentially targeting both chromosomes using different resistance genes [1,2]. On the other hand, haploid cells contain only one copy of each chromosome, and disruption of one allele can therefore produce a loss-of-function phenotype (Figure 1). Therefore, gene manipulation in haploid cells can simplify genetic analysis and provide valuable information regarding the molecular mechanisms of numerous genes and proteins. However, these haploid cells are generally limited in their life cycle.

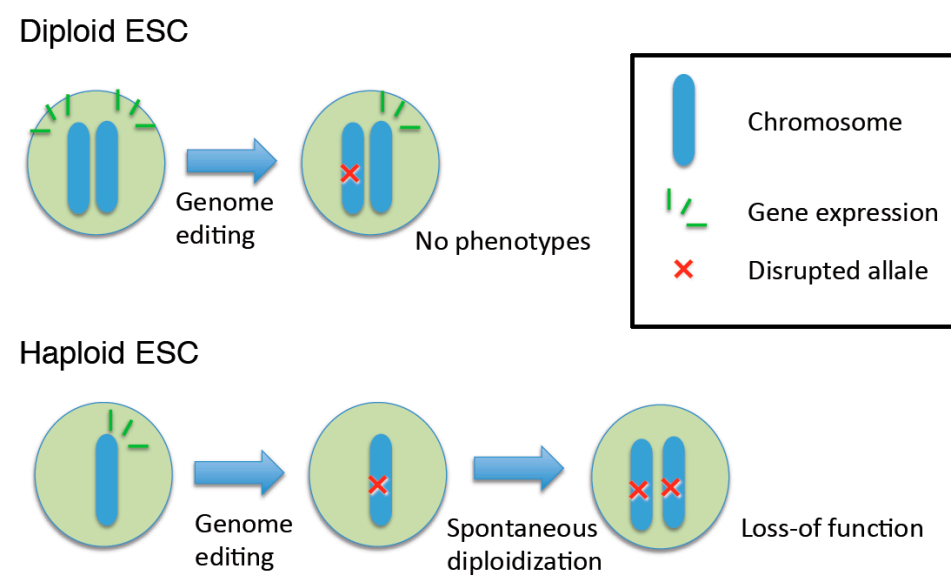

Figure 1. Haploid embryonic stem cells (ESCs) are useful for studying gene functions because disruption of a single allele can cause loss-of-function phenotypes.

In vertebrates, the first haploid cell lines were obtained more than 40 years ago from frogs [3]. Despite this, the availability of isolated vertebrate haploid cell lines is limited. Mammalian (especially human) haploid cell lines are of particular interest, because genetic screening in mammalian cells is directly applicable to medicinal and pharmacological research. Since the 1970s, several strategies have been used in attempts to produce murine haploid embryos [4-7]. Successful generation of diploid ESCs in mice [8] suggested that production of haploid ESC lines would be possible; however, the haploid cells became spontaneously diploid during ESC establishment [9]. Haploid ESC lines were recently produced from medaka fish [10]. This led to renewed attempts to generate mammalian haploid ESCs, and mouse haploid ESCs were subsequently produced by two different research groups [11,12].

\section{Derivation of Haploid ESCs}

The success in generating mouse haploid ESCs was due to advances in ESC culture systems and improvements in the purification of haploid cells using fluorescence-activated cell sorting (FACS). The first mammalian haploid ESCs were generated from parthenogenetic embryos produced by artificial activation of oocytes with ethanol [11] or strontium chloride [12] (Figure 2). Subsequently, androgenetic haploid ESCs were generated from androgenetic embryos containing only the paternal genome set $[13,14]$. Androgenetic embryos were produced by removal of the maternal pronucleus [14] or by sperm injection into enucleated oocytes [13,14] (Figure 2). Both parthenogenetic and androgenetic haploid ESCs were functional in chimeric mice, and were able to differentiate to several 
tissues in vitro [11-14]. Remarkably, the haploid cells underwent diploidization during the differentiation process both in vivo and in vitro (Figure 1). Genomic imprinting, an epigenetic phenomenon via which certain genes are expressed in a parental origin-specific manner, was partially retained in both androgenetic and parthenogenetic haploid ESCs. Androgenetic and parthogenetic ESCs could therefore be substituted for sperm [13,14] and oocytes [15], respectively. Complete haploid ESCs were generated in other species: for example, androgenetic haploid ESCs were generated in rats [16] and parthenogenetic haploid ESCs in monkeys [17].

By contrast, near-haploid cell lines have been generated from human tumors [18] and used in genetic screens to screen for human host factors involved in infection by pathogens [19-21] and the toxic effects of bacterial toxins [22-24]. This work has laid the foundation of haploid genetics in mammals today. Human haploid ESCs have not yet been reported; however, successful generation of non-primate haploid ESCs suggests that generation of human haploid ESCs may be possible in the near future.

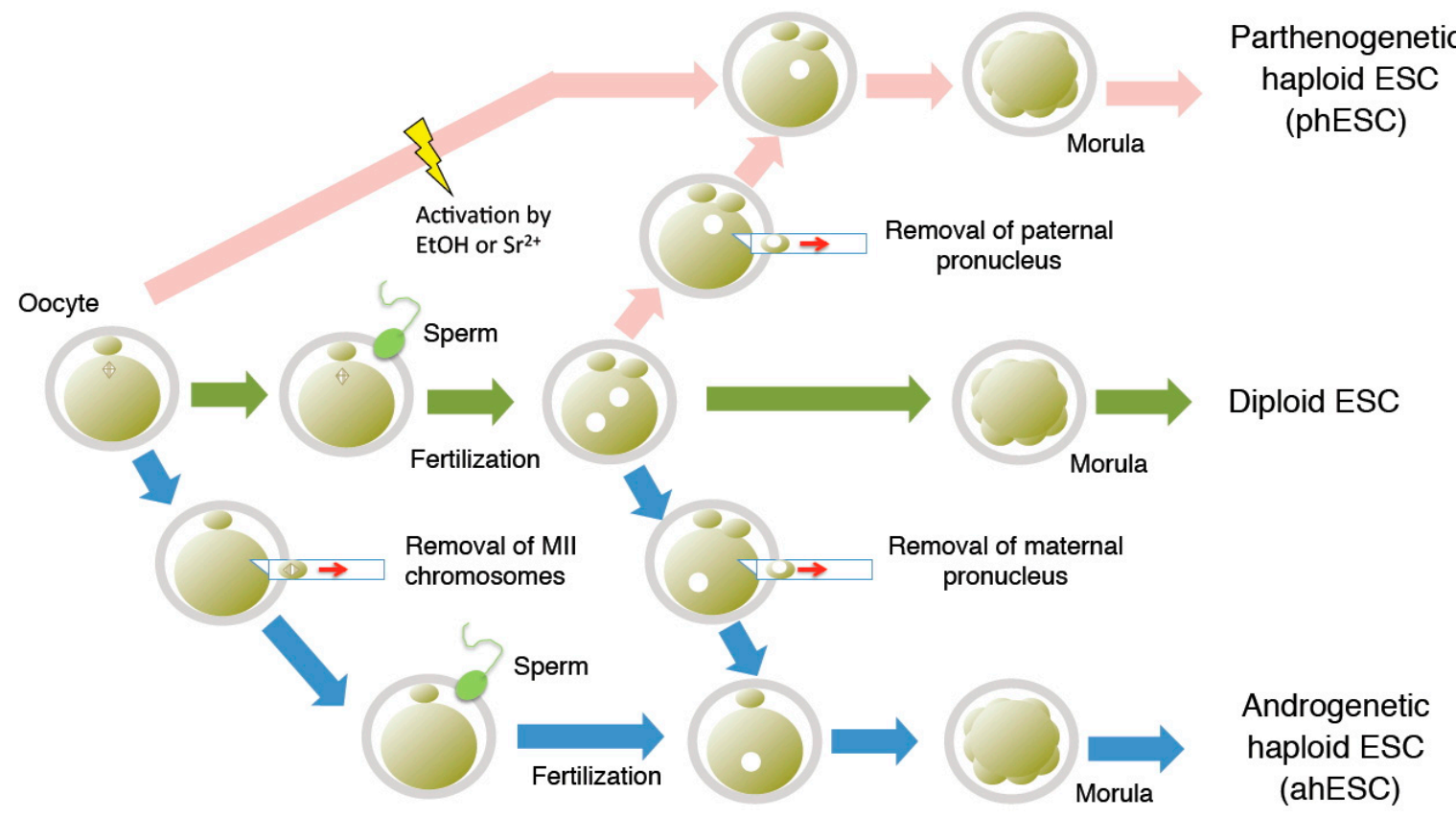

Figure 2. Generation of haploid ESCs via a range of methods. Pink arrows: generation of phESC; blue arrows: generation of ahESC; green arrows: generation of normal diploid ESC; yellow shape: artificial activation by ethanol $(\mathrm{EtOH})$ or strontium chloride $\left(\mathrm{Sr}^{2+}\right)$.

\section{Purification and Maintenance of Haploid ESCs}

Haploid ESCs tend to diploidize spontaneously due to endoreduplication or missed cytokinesis [25]. Haploid ESCs generally become completely diploid within 3 weeks. It is therefore necessary to purify cells with haploid G1 DNA content at certain intervals (Figure 3). Generally, haploid cells are purified by cell sorting after staining with Hoechst 33342, which is detected using a $375 \mathrm{~nm}$ near-ultra-violet (UV) laser. However, as most FACS equipment does not have near-UV detection, improved sorting techniques are needed to increase access to haploid ESC research.

Recently, we and others [26] developed a novel haploid purification system that used a scatter plot generated by FACS analysis with a standard $488 \mathrm{~nm}$ laser. No cell staining was required in this system. Scatter plots show forward scatter (FSC) and side scatter (SSC) and can be used to distinguish the 
physical properties of a particle, such as size and internal complexity. At G1 phase, haploid ESCs are smaller than diploid ESCs $(10.0 \pm 1.0 \mu \mathrm{m} v s .16 .5 \pm 2.2 \mu \mathrm{m}$ in diameter $)$. The novel purification method was able to distinguish haploid ESCs, as indicated by the circled population (P1, blue dots) in the scatter plot in Figure 4. Haploid G1 DNA content was confirmed in the P1 population after cell sorting. In this purification system, the most important procedure is selecting a P1 population, which is largely absent from diploid cells. Therefore, it is essential to use diploid ESCs as a negative control.

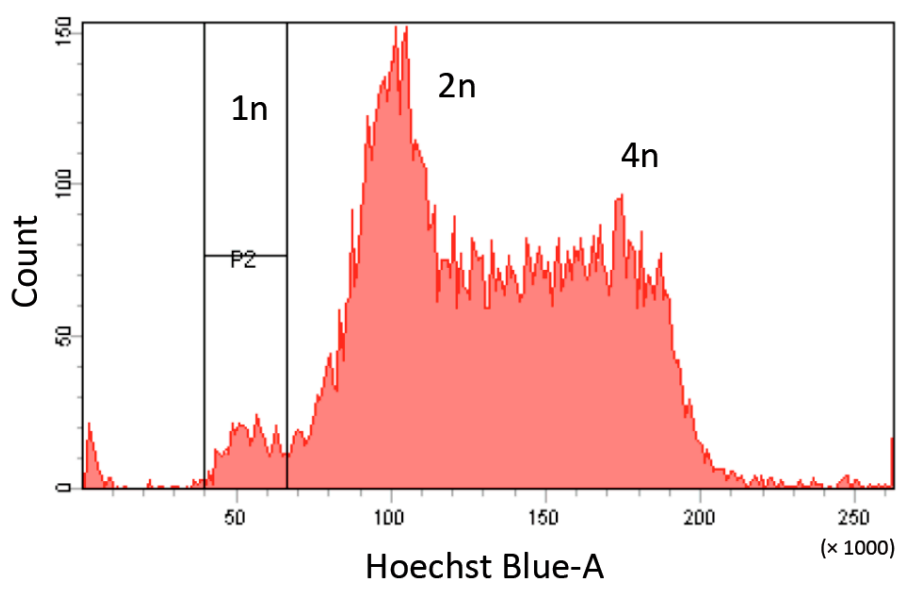

Figure 3. Identification of haploid ESCs by Hoechst 33342 staining. Cells with haploid G1 content ( $1 n$ peak) are purified by fluorescence-activated cell sorting (FACS) using a near-UV laser (375 nm).

Haploid ESC
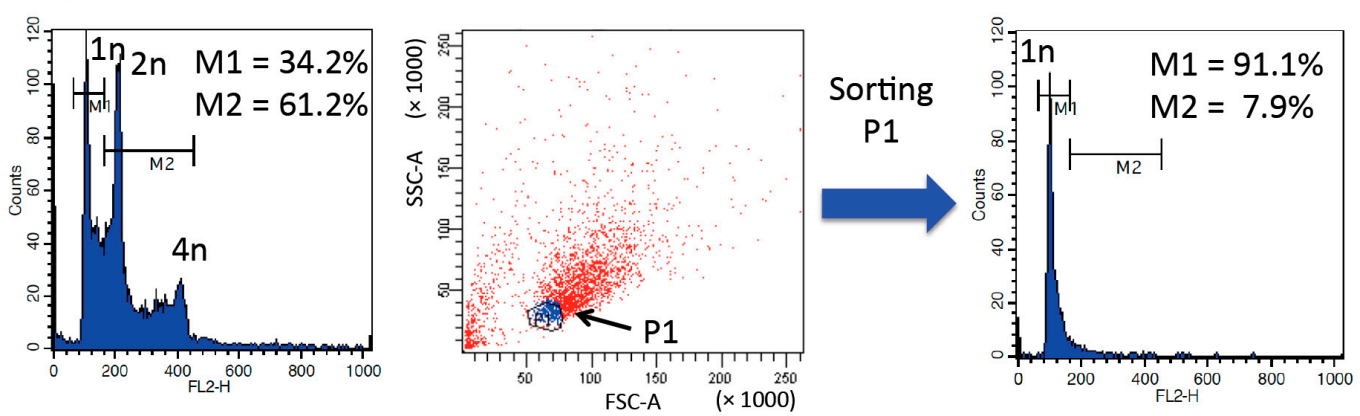

Diploid ESC
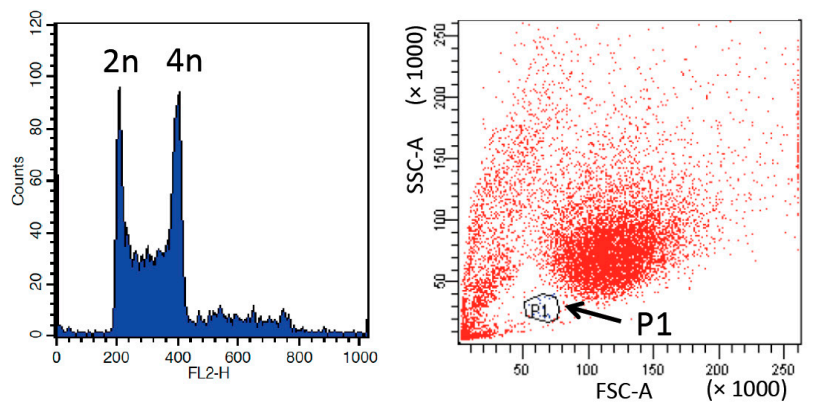

Figure 4. Purification of $1 \mathrm{n}$ peak of haploid ESCs using a forward scatter (FSC)/side scatter (SSC) scatter plot to determine cell size. Propidium iodide staining after fixation confirmed that population 1 (P1) contained haploid G1 ESCs and that the P1 population was largely absent in diploid ESCs. 
Accurate cell sorting is just one requirement for successful maintenance of a haploid ESC population. It is also important to inhibit spontaneous diploidization during daily culture of haploid ESCs. Glycogen synthase kinase (GSK) 3 and ERK inhibitors (2i) are important inhibitors of haploid ESC diploidization [25]. Stability of the haploid karyotype is increased in the naïve ground state by using 2i-supplemented ESC medium. By contrast, exit from the naïve ground state induces diploidization. For example, addition of FGF/activin to growth medium accelerates diploidization by prompting a shift from the naïve ground state to the primed state in epiblast stem cells (EpiSCs) [25]. Dosage compensation occurs in female XX diploid EpiSCs via Xist expression and heterochromatic inactivation of one $\mathrm{X}$ chromosome [27]. In these diploid cells, the ratio of X-linked (X) to autosomal gene expression (A) is 1:2. In haploid cells, the gene expression ratio of X:A is 1:1. Haploid EpiSCs, with abnormal dosage compensation might be unstable for cell survival and stimulate diploidization. Inclusion of $2 \mathrm{i}$ in growth medium is thus key to inhibiting diploidization of haploid ESCs.

Takahashi and colleagues suggested that diploidization occurred partly as a result of abnormal cell cycle regulation in haploid cells. In this process, cell division was skipped once and cells entered an extra G1/S phase [28]. To overcome this spontaneous diploid conversion, researchers applied culture medium containing a small molecular inhibitor of Weel kinase. This regulated the cell cycle by accelerating the $\mathrm{G} 2 / \mathrm{M}$ phase transition and preventing entry into the extra G1/S phase. Haploid status was maintained under these conditions, without cell sorting by FACS, for at least 4 weeks.

\section{Genome Editing in Haploid ESCs}

Haploid ESCs are valuable tools for reverse and forward genetic screens. For example, Elling and colleagues used retroviral mutagenesis to generate insertional mutants in haploid ESCs [11]. A forward genetic screen in haploid ESCs by the same authors identified Gpr107 as a key molecule involved in ricin toxicity. Such experiments might be streamlined by the application of recently-developed genome-editing techniques.

The clustered regularly interspaced short palindromic repeats (CRISPR) system, which uses the RNA-guided nuclease Cas9, is a novel genome-editing technology for mammalian cells [29-32]. This system uses a single gene encoding the Cas9 protein alongside a small guide RNA (sgRNA) complementary to the target DNA sequence [33]. The Cas9 endonuclease generates sequence-specific double-strand breaks (DSBs) at target DNAs bound to sgRNAs. The subsequent repair of the break by non-homologous end joining (NHEJ) frequently introduces mutations. The CRISPR/Cas system has been used to perform high-efficiency loss-of-function screening in mouse and human diploid cell lines [34-36]. It is also effective for gene knockout and knockin in human induced pluripotent stem cells (iPSCs) [32], suggesting that it could be used to generate disease model cell lines [37,38] and obtain precise correction of mutant genes [39-43] in human iPSCs.

Recently, we reported the use of the CRISPR/Cas system to manipulate mammalian genes in mouse haploid ESCs [44]. Haploid ESCs were co-transfected with vectors expressing Cas9 nuclease and sgRNAs targeting the Tet1, Tet2, and Tet3 genes. Simultaneous disruption of all three genes and corresponding loss-of-function was observed at high frequency (10/20 clones, 50\%) (Figure 5A). This targeting (triple knockout) efficiency in haploid ESCs is higher than that previously reported in diploid ESCs (20/96 clones, 20.8\%) [45] and in our previous unpublished study (2/20 clones, 10\%). Similar 
experiments using rat haploid ESCs [16] also mutated all three Tet genes with high efficiency (3/22 clones, 13.6\%). Large chromosome deletions can be produced by using a pair of sgRNAs. Canver and colleagues reported that cleavage of genome sequences can result in the removal of $1 \mathrm{Mb}$ of DNA from mammalian diploid cells [46]. For example, the frequency of biallelic large deletions was $2.5 \%$ (2/80 clones) for a $13.5 \mathrm{~kb}$ deletion, and $10.1 \%$ (16/158 clones) for a $15 \mathrm{~kb}$ deletion. In our study, haploid ESCs were also co-transfected with vectors expressing Cas9 and sgRNAs targeting two loci (Tet1 exons 4 and 7) [44]. This led to the deletion of a $14 \mathrm{~kb}$ chromosomal fragment (6/20 clones, $30 \%)$ or inversion of the large fragment $(2 / 20$ clones, $10 \%)$ in a substantial proportion of transfected cells (Figure 5B). Successful CRISPR/Cas-mediated knock-in was also reported in mouse haploid ESCs [47,48] (Figure 5C). Most recently, Zhong and colleagues conducted CRISPR/Cas-mediated genetic screening in mouse haploid ESCs carrying a guide RNA library [48]. Genome-edited mouse androgenetic haploid ESCs were used that could support full-term development of semi-cloned embryos upon injection into MII oocytes. The CRISPR/Cas system is therefore especially powerful for manipulation of mammalian genomes when performed in haploid ESCs. In particular, this will facilitate reverse and forward genetic screens.

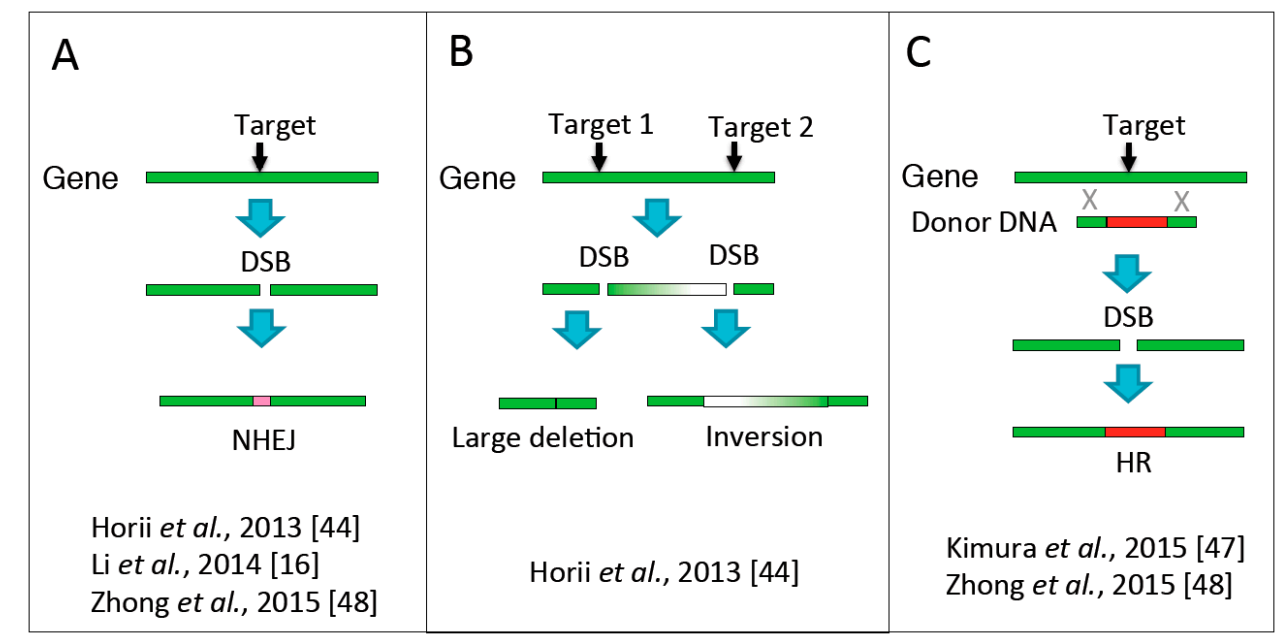

Figure 5. Genome editing in haploid ESCs using the clustered regularly interspaced short palindromic repeats (CRISPR)/Cas system. (A) Gene disruption by induction of double-stranded-break (DSB) formation followed by repair by non-homologous end joining (NHEJ); (B) deletion or inversion of large genomic regions as a result of two induced DSBs; (C) gene knock-in by homologous recombination (HR) with donor DNA.

We have to discuss potential off-target effects in haploid cells. The off-target effect involves non-specific recognition and digestion at non-targeted regions by the CRISPR/Cas system. Haploid cells contain only one copy of each chromosome; thus, disruption of one allele can lead easily to a loss-of-function phenotype. However, this is a double-edged sword because disruption of one allele on off-target site(s) can produce undesirable loss-of-function phenotypes. In several human tumor cell lines, a high frequency of off-target effects was observed [49-51], whereas a low frequency was observed in mouse and its ESC lines [45,52]. However, off-target effects can occur in any cell line. Off-target effects could be reduced in haploid cells by employing the double-nicking strategy with Cas9 nickase, which cleaves only single stranded DNA, and a pair of guide RNAs [53]. 
The major advantage of using haploid cells is the ease with which genomic manipulation and observation of phenotypes can be performed. Another attractive feature is that ESCs can be maintained as haploid cells only for the duration of editing and then allowed to become diploid (and homozygous for the introduced mutation) for further characterization. In addition, haploid ESCs exhibit pluripotency or multipotency; therefore, screened ESCs can be differentiated both in vivo and in vitro. Near-haploid tumor cell lines that have been used for haploid genetics in human do not have this feature. If human haploid ESCs are generated in the near future, tissues and organs derived from the screened ESC lines could be used for various analyses.

\section{Conclusions}

Use of the CRISPR/Cas genome-editing system in haploid ESCs allows efficient genetic manipulation of the mammalian genome. This technique provides a new platform for genetic analyses of complex biological phenomena and diseases.

\section{Acknowledgments}

This work was supported in part by grants from the Ministry of Education, Culture, Sports, Science, and Technology of Japan; the Ministry of Health, Labor, and Welfare of Japan; the National Institute of Biomedical Innovation; the Takeda Science Foundation; a grant from the Asahi Glass Foundation; and a grant from the Ichiro Kanehara Foundation.

\section{Author Contributions}

Takuro Horii and Izuho Hatada wrote the manuscript.

\section{Conflicts of Interest}

The authors declare no conflicts of interest.

\section{References}

1. Mortensen, R.M.; Zubiaur, M.; Neer, E.J.; Seidman, J.G. Embryonic stem cells lacking a functional inhibitory G-protein subunit $(\alpha$ i2) produced by gene targeting of both alleles. Proc. Natl. Acad. Sci. USA 1991, 88, 7036-7040.

2. Milstone, D.S.; Bradwin, G.; Mortensen, R.M. Simultaneous Cre catalyzed recombination of two alleles to restore neomycin sensitivity and facilitate homozygous mutations. Nucleic Acids Res. 1999, 27, e10, doi:10.1093/nar/27.15.e10.

3. Freed, J.J.; Mezger-Freed, L. Stable haploid cultured cell lines from frog embryos. Proc. Natl. Acad. Sci. USA 1970, 65, 337-344.

4. Graham, C.F. The effect of cell size and DNA content on the cellular regulation of DNA synthesis in haploid and diploid embryos. Exp. Cell Res. 1966, 43, 13-19.

5. Modlinski, J.A. Haploidmouse embryos obtained by microsurgical removal of one pronucleus. J. Embryol. Exp. Morphol. 1975, 33, 897-905. 
6. Tarkowski, A.K.; Rossant, J. Haploidmouse blastocysts developed from bisected zygotes. Nature 1976, 259, 663-665.

7. Kaufman, M.H. Chromosome analysis of early postimplantation presumptive haploid parthenogenetic mouse embryos. J. Embryol. Exp. Morphol. 1978, 45, 85-91.

8. Evans, M.J.; Kaufman, M.H. Establishment in culture of pluripotential cells from mouse embryos. Nature 1981, 292, 154-156.

9. Kaufman, M.H.; Robertson, E.J.; Handyside, A.H.; Evans, M.J. Establishment of pluripotential cell-lines from haploid mouse embryos. J. Embryol. Exp. Morph. 1983, 73, 249-261.

10. Yi, M.; Hong, N.; Hong, Y. Generation of medaka fish haploid embryonic stem cells. Science 2009, 326, 430-433.

11. Elling, U.; Taubenschmid, J.; Wirnsberger, G.; O’Malley, R.; Demers, S.P.; Vanhaelen, Q.; Shukalyuk, A.I.; Schmauss, G.; Schramek, D.; Schnuetgen, F.; et al. Forward and reverse genetics through derivation of haploid mouse embryonic stem cells. Cell Stem Cell 2011, 9, 563-574.

12. Leeb, M.; Wutz, A. Derivation of haploid embryonic stem cells from mouse embryos. Nature 2011, 479, 131-134.

13. Li, W.; Shuai, L.; Wan, H.; Dong, M.; Wang, M.; Sang, L.; Feng, C.; Luo, G.Z.; Li, T.; Li, X.; et al. Androgenetic haploid embryonic stem cells produce live transgenic mice. Nature 2012, 490, 407-411.

14. Yang, H.; Shi, L.; Wang, B.A.; Liang, D.; Zhong, C.; Liu, W.; Nie, Y.; Liu, J.; Zhao, J. Gao, X.; et al. Generation of genetically modified mice by oocyte injection of androgenetic haploid embryonic stem cells. Cell 2012, 149, 605-617.

15. Wan, H.; He, Z.; Dong, M.; Gu, T.; Luo, G.Z.; Teng, F.; Xia, B.; Li, W.; Feng, C.; Li, X.; et al. Parthenogenetic haploid embryonic stem cells produce fertile mice. Cell Res. 2013, 23, 1330-1333.

16. Li, W.; Li, X.; Li, T.; Jiang, M.G.; Wan, H.; Luo, G.Z.; Feng, C.; Cui, X.; Teng, F.; Yuan, Y.; et al. Genetic modification and screening in rat using haploid embryonic stem cells. Cell Stem Cell 2013, 14, 404-414.

17. Yang, H.; Liu, Z.; Ma, Y.; Zhong, C.; Yin, Q.; Zhou, C.; Shi, L.; Cai, Y.; Zhao, H.; Wang, H.; et al. Generation of haploid embryonic stem cells from Macaca fascicularis monkey parthenotes. Cell Res. 2013, 23, 1187-1200.

18. Kotecki, M.; Reddy, P.S.; Cochran, B.H. Isolation and characterization of a near-haploid human cell line. Exp. Cell Res. 1999, 252, 273-280.

19. Carette, J.E.; Guimaraes, C.P.; Varadarajan, M.; Park, A.S.; Wuethrich, I.; Godarova, A.; Kotecki, M.; Cochran, B.H.; Spooner, E.; Ploegh, H.L.; et al. Haploid genetic screens in human cells identify host factors used by pathogens. Science 2009, 326, 1231-1235.

20. Carette, J.E.; Raaben, M.; Wong, A.C.; Herbert, A.S.; Obernosterer, G.; Mulherkar, N.; Kuehne, A.I.; Kranzusch, P.J.; Griffin, A.M.; Ruthel, G.; et al. Ebola virus entry requires the cholesterol transporter Niemann-Pick C1. Nature 2011, 477, 340-343.

21. Rosmarin, D.M.; Carette, J.E.; Olive, A.J.; Starnbach, M.N.; Brummelkamp, T.R.; Ploegh, H.L. Attachment of Chlamydia trachomatis L2 to host cells requires sulfation. Proc. Natl. Acad. Sci. USA 2012, 109, 10059-10064. 
22. Reiling, J.H.; Clish, C.B.; Carette, J.E.; Varadarajan, M.; Brummelkamp, T.R.; Sabatini, D.M. A haploid genetic screen identifies the major facilitator domain containing 2A (MFSD2A) transporter as a key mediator in the response to tunicamycin. Proc. Natl. Acad. Sci. USA 2011, 108, 11756-11765.

23. Reiling, J.H.; Olive, A.J.; Sanyal, S.; Carette, J.E.; Brummelkamp,T.R.; Ploegh, H.L.; Starnbach, M.N.; Sabatini, D.M. ACREB3-ARF4 signalling pathway mediates the response to Golgi stress and susceptibility to pathogens. Nat. Cell Biol. 2013, 15, 1473-1485.

24. Birsoy, K.; Wang, T.; Possemato, R.; Yilmaz, O.H.; Koch, C.E.; Chen, W.W.; Hutchins, A.W.; Gultekin, Y.; Peterson, T.R.; Carette, J.E.; et al. MCT1-mediated transport of a toxic molecule is an effective strategy for targeting glycolytic tumors. Nat. Genet. 2013, 45, 104-108.

25. Leeb, M.; Perry, A.C.; Wutz, A. Establishment and use of mouse haploid ES cells. Curr. Protoc. Mouse Biol. 2015, 5, 155-185.

26. Leeb, M.; Walker, R.; Mansfield, B.; Nichols, J.; Smith, A.; Wutz, A. Germline potential of parthenogenetic haploid mouse embryonic stem cells. Development 2012, 139, 3301-3305.

27. Guo, G.; Yang, J.; Nichols, J.; Hall, J.S.; Eyres, I.; Mansfield, W.; Smith, A. Klf4 reverts developmentally programmed restriction of ground state pluripotency. Development 2009, 136, 1063-1069.

28. Takahashi, S.; Lee, J.; Kohda, T.; Matsuzawa, A.; Kawasumi, M.; Kanai-Azuma, M.; Kaneko-Ishino, T.; Ishino, F. Induction of the G2/M transition stabilizes haploid embryonic stem cells. Development 2014, 141, 3842-3847.

29. Cho, S.W.; Kim, S.; Kim, J.M.; Kim, J.S. Targeted genome engineering in human cells with the Cas9 RNA-guided endonuclease. Nat. Biotechnol. 2013, 31, 230-232.

30. Cong, L.; Ran, F.A.; Cox, D.; Lin, S.; Barretto, R.; Habib, N.; Hsu, P.D.; Wu, X.; Jiang, W.; Marraffini, L.A.; et al. Multiplex genome engineering using CRISPR/Cas systems. Science 2013, 339, 819-823.

31. Jinek, M.; East, A.; Cheng, A.; Lin, S.; Ma, E.; Doudna, J. RNA-programmed genome editing in human cells. eLife 2013, 2, e00471, doi:10.7554/eLife.00471.

32. Mali, P.; Yang, L.; Esvelt, K.M.; Aach, J.; Guell, M.; DiCarlo, J.E.; Norville, J.E.; Church, G.M. RNA-guided human genome engineering via Cas9. Science 2013, 339, 823-826.

33. Jinek, M.; Chylinski, K.; Fonfara, I.; Hauer, M.; Doudna, J.A.; Charpentier, E. A programmable dual-RNA-guided DNA endonuclease in adaptive bacterial immunity. Science 2012, 337, 816-821.

34. Koike-Yusa, H.; Li, Y.; Tan, E.P.; Velasco-Herrera, M.D.C.; Yusa, K. Genome-wide recessive genetic screening in mammalian cells with a lentiviral CRISPR-guide RNA library. Nat. Biotechnol. 2014, 32, 267-273.

35. Shalem, O.; Sanjana, N.E.; Hartenian, E.; Shi, X.; Scott, D.A.; Mikkelsen, T.S.; Heckl, D.; Ebert, B.L.; Root, D.E.; Doench, J.G.; et al. Genome-scale CRISPR-Cas9 knockout screening in human cells. Science 2014, 343, 84-87.

36. Wang, T.; Wei, J.J.; Sabatini, D.M.; Lander, E.S. Genetic screens in human cells using the CRISPR-Cas9 system. Science 2014, 343, 80-84.

37. Horii, T.; Tamura, D.; Morita, S.; Kimura, M.; Hatada, I. Generation of an ICF syndrome model by efficient genome editing of human induced pluripotent stem cells using the CRISPR system. Int. J. Mol. Sci. 2013, 14, 19774-19781. 
38. An, M.C.; O’Brien, R.N.; Zhang, N.; Patra, B.N.; de La Cruz, M.; Ray A.; Ellerby, L.M. Polyglutamine disease modeling: Epitope based screen for homologous recombination using CRISPR/Cas9 system. PLoS Curr. 2014, 6, doi:10.1371/currents.hd.0242d2e7ad72225efa72 f6964589369a.

39. Xie, F.; Ye, L.; Chang, J.C.; Beyer, A.I.; Wang, J.; Muench, M.O.; Kan, Y.W. Seamless gene correction of $\beta$-thalassemia mutations in patient-specific iPSCs using CRISPR/Cas9 and piggyback. Genome Res. 2014, 24, 1526-1533.

40. Li, H.L.; Fujimoto, N.; Sasakawa, N.; Shirai, S.; Ohkame, T.; Sakuma, T.; Tanaka, M.; Amano, N.; Watanabe, A.; Sakurai, H.; et al. Precise correction of the dystrophin gene in duchenne muscular dystrophy patient induced pluripotent stem cells by TALEN and CRISPR-Cas9. Stem Cell Rep. 2015, 4, 143-154.

41. Ousterout, D.G.; Kabadi, A.M.; Thakore, P.I.; Majoros, W.H.; Reddy, T.E.; Gersbach, C.A. Multiplex CRISPR/Cas9-based genome editing for correction of dystrophin mutations that cause Duchenne muscular dystrophy. Nat. Commun. 2015, 6, 6244, doi:10.1038/ncomms 7244.

42. Song, B.; Fan, Y.; He, W.; Zhu, D.; Niu, X.; Wang, D.; Ou, Z.; Luo, M.; Sun, X. Improved hematopoietic differentiation efficiency of gene-corrected beta-thalassemia induced pluripotent stem cells by CRISPR/Cas9 system. Stem Cells Dev. 2015, 24, 1053-1065.

43. Huang, X.; Wang, Y.; Yan, W.; Smith, C.; Ye, Z.; Wang, J.; Gao, Y.; Mendelsohn, L.; Cheng, L. Production of gene-corrected adult $\beta$ globin protein in human erythrocytes differentiated from patient iPSCs after genome editing of the sickle point mutation. Stem Cells 2015, 33, 1470-1479.

44. Horii, T.; Morita, S.; Kimura, M.; Kobayashi, R.; Tamura, D.; Takahashi, R.U.; Kimura, H.; Suetake, I.; Ohata, H.; Okamoto, K.; et al. Genome engineering of mammalian haploid embryonic stem cells using the Cas9/RNA system. PeerJ 2013, 1, e230, doi:10.7717/peerj.230.

45. Wang, H.; Yang, H.; Shivalila, C.S.; Dawlaty, M.M.; Cheng, A.W.; Zhang, F.; Jaenisch, R. One-step generation of mice carrying mutations in multiple genes by CRISPR/Cas-mediated genome engineering. Cell 2013, 153, 910-918.

46. Canver, M.C.; Bauer, D.E.; Dass, A.; Yien, Y.Y.; Chung, J.; Masuda, T.; Maeda, T.; Paw, B.H.; Orkin, S.H. Characterization of genomic deletion efficiency mediated by clustered regularly interspaced palindromic repeats (CRISPR)/Cas9 nuclease system in mammalian cells. J. Biol. Chem. 2014, 289, 21312-21324.

47. Kimura, Y.; Oda, M.; Nakatani, T.; Sekita, Y.; Monfort, A.; Wutz, A.; Mochizuki, H.; Nakano, T. CRISPR/Cas9-mediated reporter knock-in in mouse haploid embryonic stem cells. Sci. Rep. 2015, 5, 10710, doi:10.1038/srep10710.

48. Zhong, C.; Yin, Q.; Xie, Z.; Bai, M.; Dong, R.; Tang, W.; Xing, Y.H.; Zhang, H.; Yang, S.; Chen, L.L.; et al. CRISPR-Cas9-Mediated Genetic Screening in Mice with Haploid Embryonic Stem Cells Carrying a Guide RNA Library. Cell Stem Cell 2015, 17, 221-232.

49. Fu, Y.; Foden, J.A.; Khayter, C.; Maeder, M.L.; Reyon, D.; Joung, J.K.; Sander, J.D. High-frequency off-target mutagenesis induced by CRISPR-Cas nucleases in human cells. Nat. Biotechnol. 2013, 31, 822-826.

50. Hsu, P.D.; Scott, D.A.; Weinstein, J.A.; Ran, F.A.; Konermann, S.; Agarwala, V.; Li, Y.; Fine, E.J.; Wu, X.; Shalem, O.; et al. DNA targeting specificity of RNA-guided Cas9 nucleases. Nat. Biotechnol. 2013, 31, 827-832. 
51. Pattanayak, V.; Lin, S.; Guilinger, J.P.; Ma, E.; Doudna, J.A.; Liu, D.R. High-throughput profiling of off-target DNA cleavage reveals RNA-programmed Cas9 nuclease specificity. Nat. Biotechnol. 2013, 31, 839-843.

52. Yang, H.; Wang, H.; Shivalila, C.S.; Cheng, A.W.; Shi, L.; Jaenisch, R. One-Step generation of mice carrying reporter and conditional alleles by CRISPR/Cas-mediated genome engineering. Cell 2013, 154, 1370-1379.

53. Ran, F.A.; Hsu, P.D.; Lin, C.Y.; Gootenberg, J.S.; Konermann, S.; Trevino, A.E.; Scott, D.A.; Inoue, A.; Matoba, S.; Zhang, Y.; Zhang, F. Double nicking by RNA-guided CRISPR Cas9 for enhanced genome editing specificity. Cell 2013, 154, 1380-1389.

(C) 2015 by the authors; licensee MDPI, Basel, Switzerland. This article is an open access article distributed under the terms and conditions of the Creative Commons Attribution license (http://creativecommons.org/licenses/by/4.0/). 